\title{
Permutation Labeling for Some Shadow Graphs
}

\author{
J. Shiama \\ Assistant Professor \\ Department Of Mathematics \\ Cms College Of Science And Commerce, \\ Coimbatore -641049 , Tamilnadu, India
}

\begin{abstract}
A permutation labeling of a graph $\mathrm{G}$ is a bijective assignment of labels from $\{1,2,3, \ldots . p\}$ to the vertices of $G$ such that when each edge of $G$ has assigned a weight defined by the number of permutations of $f(u)$ things taken $f(v)$ at a time. Such a labeling $f$ is called permutation labeling of G. A graph which admits permutation labeling is called permutation graphs. In this paper I proved that the shadow graphs of path $P_{n}$, star $K_{1, n}$ and path union of shadow graphs of cycle $C_{n}$ are permutation graphs. Further I proved that the split graphs of path $P_{n}$ and star $K_{1, n}$ are permutation graphs.
\end{abstract}

Keywords: Permutation labeling, Shadow graph, Split graph, path union.

\section{INTRODUCTION}

Throughout this paper we consider simple, finite, connected and undirected graph $\mathbf{G}=(\mathbf{V}(\mathbf{G}), \mathbf{E}(\mathbf{G}))$ with p vertices and $q$ edges. $\mathrm{G}$ is also called a $(\mathrm{p}, \mathrm{q})$ graph. For standard terminology and notation we follow Haray[4] and Gary Chartrand and Pinz zhang[6]. For a detailed survey on graph labeling we refer Gallian [5] .The brief summary of definitions and other information which are necessary for the present investigation are given below. "Graph labeling" at its heart, is a strong communication between" number theory" [3] and "structure graphs" $[2,4,6]$. In this paper, permutation labeling of graphs are introduced and studied.

Graph labeling, where the vertices and edges are assigned real values or subsets of a set are subject to certain conditions, have often been motivated by their utility to various applied fields and their intrinsic mathematical interest ( logical- mathematical).Graph labeling were first introduced in the mid sixties

An enormous body of literature has grown around the subject, especially in the lat thirty years or so, and is still getting embellished due to increasing number of application driven concepts. Permutations play a major role in combinatorial problems. The new labeling introduced in this paper is a logical-mathematical attempt.

Definition 1.1. A (p, q) graph $G(V, E)$ is said to be a permutation graph [7] if there exists a bijection $\mathrm{f}: \mathrm{V}(\mathrm{G}) \rightarrow\{$
$1,2,3, \ldots, p\}$ such that the induced edge function $\mathrm{g}_{\mathrm{f}}: \mathrm{E}(\mathrm{G}) \rightarrow$ $\mathrm{N}$ defined as $\mathrm{g}_{\mathrm{f}}(\mathrm{uv})=\left\{\begin{array}{l}f(u) p_{f(v)} \text { if } f(u)>f(v) \\ f(v) p_{f(u)} \text { if } f(v)>f(u)\end{array}\right.$

is injective, where ${ }^{f(u)} P_{f(v)}$ denotes the number of permutations of $f(u)$ things taken $f(v)$ at a time. Such a labeling $f$ is called permutation labeling of $G$.

Definition 1.2. The shadow graph $\mathrm{D}_{2}(\mathrm{G})$ of a connected graph $\mathrm{G}$ is constructed by taking two copies of $\mathrm{G}$ say $\mathrm{G}$ ' and G' join each vertex $v$ ' in $G$; to the neighbors of the corresponding vertex v" in G".

Definition 1.3. For a graph $G$ the split graph is obtained by adding to each vertex $\mathrm{v}$ a new vertex such that $\mathrm{v}$ ' is adjacent to every vertex that is adjacent to $\mathrm{v}$ in $\mathrm{G}$. The resultant graph is denoted as $\operatorname{spl}(\mathrm{G})$

Definition 1.4.Let $G_{1}, G_{2}, \ldots . . G_{n}, n \geq 2$ be $n$ copies of a fixed graph $\mathrm{G}$. The graph $\mathrm{G}$ obtained by adding an edge between $\mathrm{G}_{\mathrm{i}}$ and $\mathrm{Gi}_{+1}$ for $\mathrm{i}=1,2, \ldots \ldots \mathrm{n}-1$ is called path union of G.[7]

\section{MAIN RESULTS}

\section{PERMUTATIONS LABELING OF SOME SHADOW GRAPHS OF PATHS, CYCLES AND STAR GRAPHS}

Theorem 2.1: $\mathrm{D}_{2}\left(\mathrm{P}_{\mathrm{n}}\right)$ admits a permutation labeling.

Proof: Consider two copies of $P_{n}$. Let $v_{1}, v_{2}, \ldots, v_{n}$ be the vertices of the first copy of $P_{n}$ and $v_{1^{\prime}}, v_{2^{\prime}}, \ldots, v_{n^{\prime}}$ be the vertices of the second copy of $P_{n}$. Let $G$ be the shadow graph $D_{2}\left(P_{n}\right)$. Then $|V(G)|=2 n$ and $|E(G)|=4(n-1)$. To define $f$ : $\mathrm{V}(\mathrm{G}) \rightarrow\{1,2, \ldots, 2 \mathrm{n}\}$

Case (i) $n$ is even, $n \geq 2$

$\mathrm{f}\left(\mathrm{v}_{\mathrm{i}}\right)=\mathrm{i} \quad(1 \leq \mathrm{i} \leq \mathrm{n})$

$\mathrm{f}\left(\mathrm{u}_{\mathrm{i}}\right)=4+\mathrm{i} \quad(1 \leq \mathrm{i} \leq \mathrm{n})$ 
Case (ii) $n$ is odd , $n \geq 3$

$\mathrm{f}\left(\mathrm{v}_{\mathrm{i}}\right)=\mathrm{i} \quad(1 \leq \mathrm{i} \leq \mathrm{n})$

$f\left(u_{i}\right)=3+i \quad(1 \leq i \leq n)$

Hence $\mathrm{D}_{2}\left(\mathrm{P}_{\mathrm{n}}\right)$ is a permutation labeling

Illustration: 2.2: The Permutation labeling of the graph $\mathrm{D}_{2}\left(\mathrm{P}_{3}\right)$ is shown in Figure-1

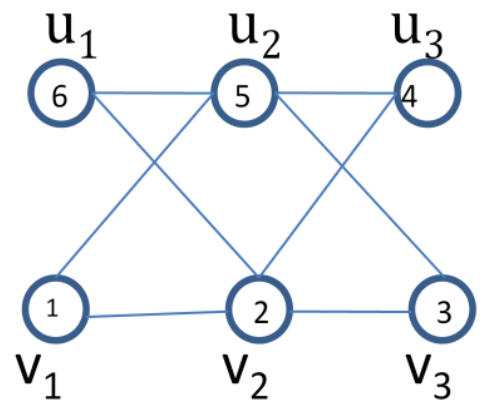

Fig-1: The Permutation labeling of the graph $D_{2}\left(P_{3}\right)$

Illustration:2.3: The Permutation labeling of the graph $\mathrm{D}_{2}\left(\mathrm{P}_{7}\right)$ is shown in Figure-2

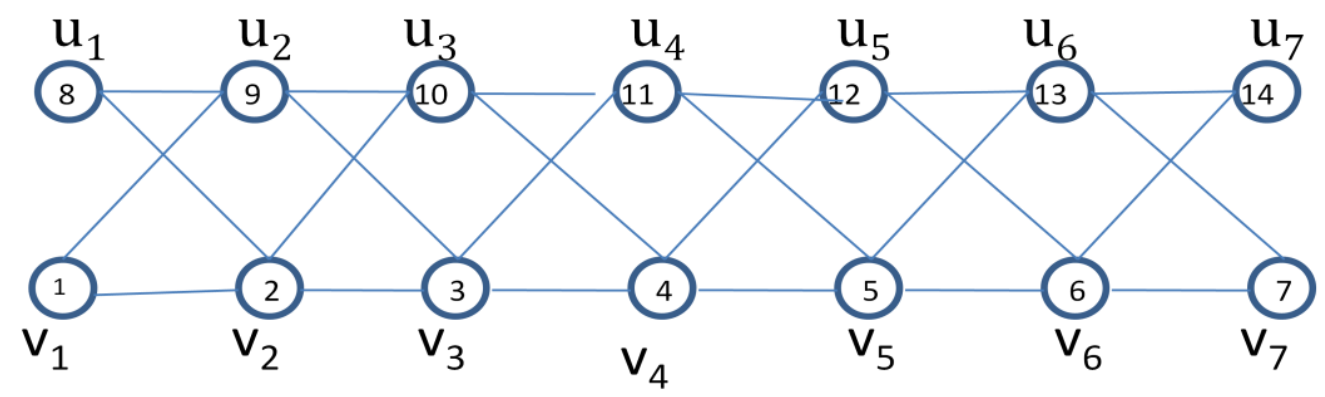

Fig-2: The Permutation labeling of the graph $D_{2}\left(P_{7}\right)$

Theorem 2.4: $\quad D_{2}\left(K_{1, n}\right)$ admits a permutation labeling.

$$
\begin{aligned}
& f(v)=1 \\
& f(v \cdot)=2 \\
& f\left(v_{i}^{\prime}\right)=6+i \quad(1 \leq i \leq n)
\end{aligned}
$$
v'respective apex vertices. Let $G=D_{2}\left(K_{1, n}\right)$. Then $|V(G)|=$ $2 n+2$ and $|E(G)|=4 n$. To define

$\mathrm{V}(\mathrm{G}) \rightarrow\{1,2, \ldots, 2 \mathrm{n}+2\}$ as follows.

$\mathrm{f}\left(\mathrm{v}_{\mathrm{i}}\right)=2+\mathrm{i} \quad(1 \leq \mathrm{i} \leq \mathrm{n})$

$\mathrm{f}(\mathrm{v})=1$

$\mathrm{f}(\mathrm{v})=$,

$6+\mathrm{i} \quad(1 \leq \mathrm{i} \leq \mathrm{n})$

Hence $\mathrm{D}_{2}\left(\mathrm{~K}_{1, \mathrm{n}}\right)$ is a permutation graph.

Illustration:2.5: Permutation labeling of the graph $\mathrm{D}_{2}\left(\mathrm{~K}_{1,4}\right)$ is shown in Figure-3. 


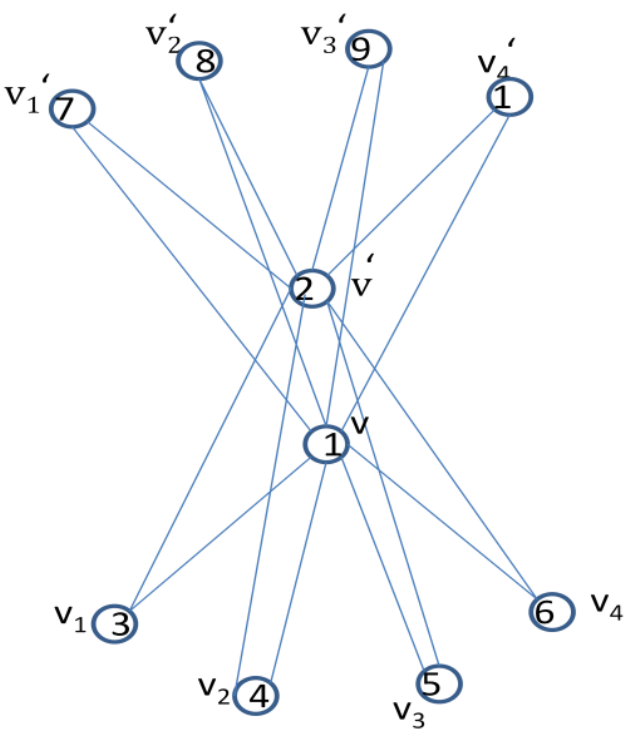

Fig-3: Permutation labeling of the graph $D_{2}\left(K_{1,4}\right)$
Proof: Let $\mathbf{D}_{2}\left(\mathrm{C}_{\mathrm{n}}\right)$ be the shadow graph of cycle. Let $\mathrm{G}$ be the path union of $k$ copies $G_{1}, G_{2}, \ldots ., G_{k}$ of shadow graph $\mathbf{D}_{2}\left(C_{n}\right)$. Let $G_{1}{ }^{\prime}, G_{2}{ }^{\prime}, \ldots \ldots \ldots G_{k}$ ' be the first copy of cycle $C_{n}$ and $G_{1}$ “", $G_{2}$ ”, , ........ $G_{k}$ ” be the second copy of cycle $C_{n}$ in $\mathbf{D}_{2}\left(C_{n}\right)$. Let us denote the successive vertices of $\mathrm{G}_{1}$ 'by $\mathrm{u}_{\mathrm{i} 1}{ }^{\prime}, \mathrm{u}_{\mathrm{i} 2}$ ',$\ldots \ldots \mathrm{u}_{\mathrm{in}}$ ' and $\mathrm{G}_{\mathrm{i}}$ " by $\mathrm{u}_{\mathrm{i} 1}$ " $, \mathrm{u}_{\mathrm{i} 2}{ }^{\prime}, \ldots \ldots \mathrm{u}_{\mathrm{in}}$ ". Let $e_{i=} u_{i 1}$ " $u_{(i+1)}$ " be the edge joining $G_{i}$ and $G_{i+1}$ for $i=1,2$, $\ldots, k$ - 1 . Define $f: V(G) \rightarrow\{1,2, \ldots, p\}$ by

$\mathrm{f}\left(\mathrm{u}_{1 \mathrm{j}^{\prime}}\right)=\mathrm{i}, \quad 1 \leq \mathrm{i} \leq \mathrm{n}$

$\mathrm{f}\left(\mathrm{u}_{1 \mathrm{j},}\right)=\mathrm{I}+\mathrm{n}, \quad 1 \leq \mathrm{i} \leq \mathrm{n}$

$\mathrm{f}\left(\mathrm{u}_{2 \mathrm{j}}\right)=\mathrm{i}+2 \mathrm{n}, \quad 1 \leq \mathrm{i} \leq \mathrm{n}$

$\mathrm{f}\left(\mathrm{u}_{2 \mathrm{j}}\right)=\mathrm{i}+3 \mathrm{n}, \quad 1 \leq \mathrm{i} \leq \mathrm{n}$

\section{Illustration:2.7:}

The permutation labeling of a graph $\mathbf{G}$ obtained by joining two copies of shadow graphs $\mathbf{D}_{2}\left(\mathrm{C}_{4}\right)$ by a path of arbitrary length is shown in figure-4

Theorem 2.6: The path union of $k$ copies of $D_{2}\left(c_{n}\right)$ is a permutation graph.

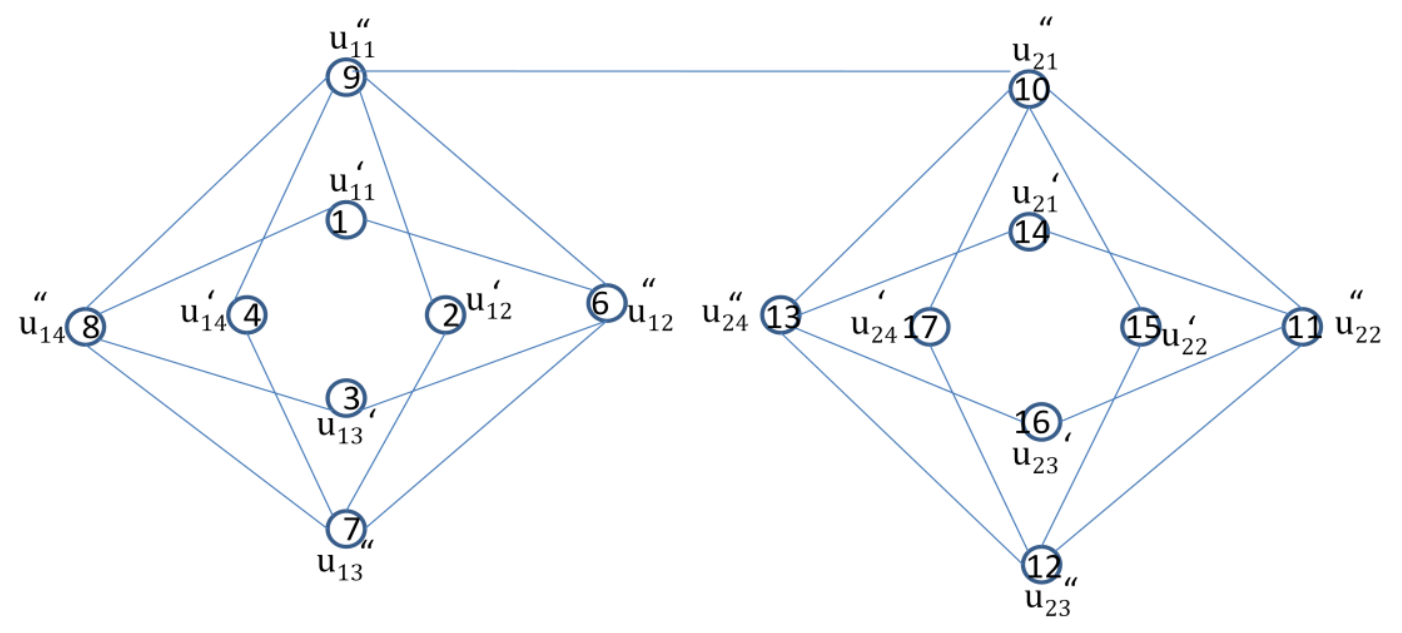

Fig-4:The permutation labeling of a graph $G$ obtained by joining two copies of shadow graphs $\mathrm{D}_{2}\left(\mathrm{C}_{4}\right)$ by a path of arbitrary length

Theorem 2.8: A graph $\mathbf{G}$ obtained by joining two copies of shadow graphs $\mathbf{D}_{\mathbf{2}}\left(\mathrm{C}_{\mathrm{n}}\right)$ by a path of arbitrary length is a permutation graph. graphs $\mathbf{D}_{\mathbf{2}}\left(\mathrm{C}_{\mathrm{n}}\right)$ by a path $\mathrm{P}_{\mathrm{k}}$ of length $\mathrm{k}-1$. Let $\mathrm{u}_{1}{ }^{,}, \mathrm{u}_{2}{ }^{\prime}, \ldots \mathrm{u}$ ${ }_{k}$ be the vertices of $C_{n}$ ' and $u_{1}{ }^{\prime}, u_{2}{ }^{\prime}, \ldots . u_{k}$ "be the vertices of $\mathrm{C}_{\mathrm{n}}$ "

Proof: Let graphs $\mathbf{D}_{\mathbf{2}}\left(\mathrm{C}_{\mathrm{n}}\right)$ be the shadow graph of cycle $\mathrm{C}_{\mathrm{n}}$ .Let $\mathbf{G}$ be a graph obtained by joining two copies of shadow 

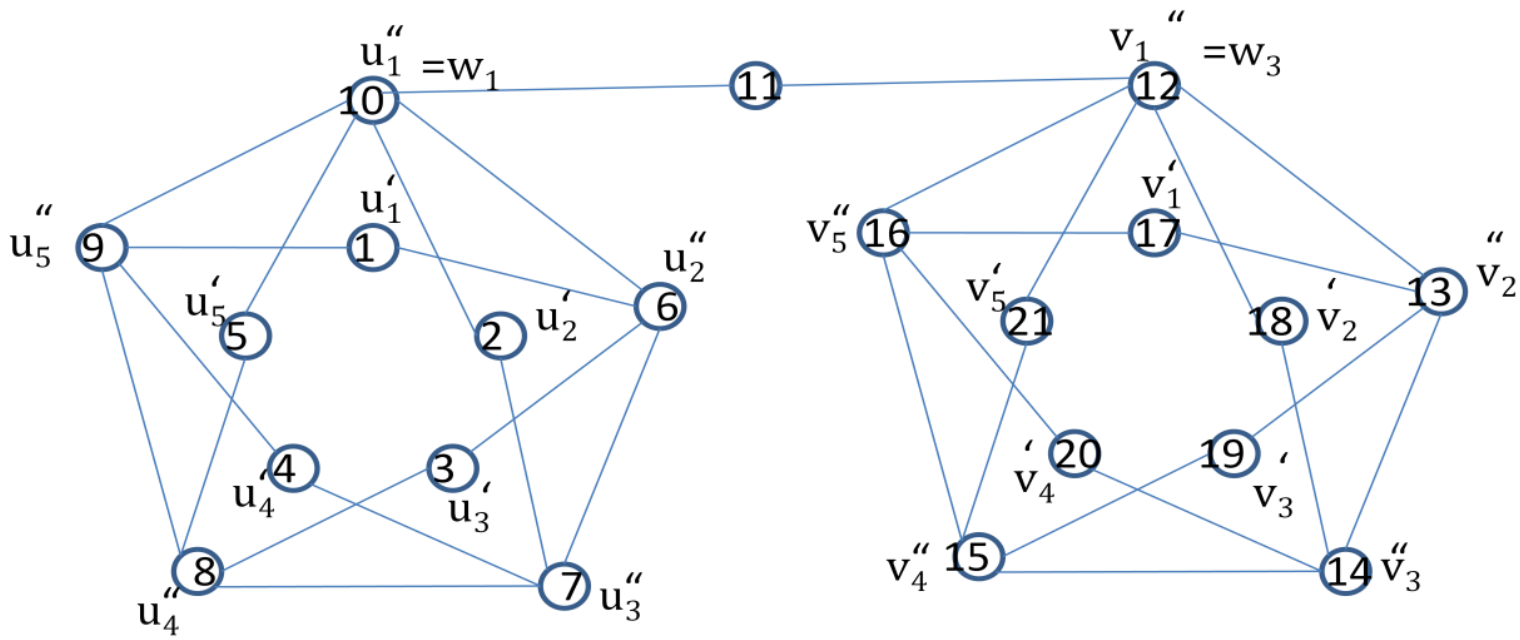

Fig-5: The permutation labeling of a graph $G$ obtained by joining two copies of shadow graphs $D_{2}\left(C_{5}\right)$ by a path of arbitrarylength

in first copy of $\mathbf{D}_{\mathbf{2}}\left(\mathrm{C}_{\mathrm{n}}\right)$. Let $\mathrm{v}_{1}, \mathrm{v}_{2}, \ldots . \mathrm{v}_{\mathrm{k}}{ }^{\prime}$ be the vertices of $\mathrm{C}_{\mathrm{n}}$ ' and $\mathrm{v}_{1}{ }^{\prime}, \mathrm{v}_{2}, \ldots . \mathrm{v}_{\mathrm{k}}{ }^{\prime}$ be the vertices of $\mathrm{C}_{\mathrm{n}}{ }^{\prime}$ in the second copy of $\mathbf{D}_{2}\left(C_{n}\right)$ in G. Let $w_{1}, w_{2}, \ldots w_{k}$ be the vertices of path $\mathrm{P}_{\mathrm{k}}$ with $\mathrm{u}_{1}{ }^{\prime}=\mathrm{w}_{1}$ and $\mathrm{v}_{1} "=\mathrm{w}_{\mathrm{k}}$. We note that $\mathrm{V}(\mathrm{G}) \mid=4 \mathrm{n}+\mathrm{k}-2$ and $|\mathrm{E}(\mathrm{G})|=8 \mathrm{n}+\mathrm{k}-3$.

To define $f: V(G) \rightarrow\{1,2, \ldots, p\}$ by

$\mathrm{f}\left(\mathrm{u}_{\mathrm{i}}\right)=\mathrm{i}, \quad 1 \leq \mathrm{i} \leq \mathrm{n}$

$\mathrm{f}\left(\mathrm{u}_{\mathrm{i}}{ }^{\prime \prime}\right)=\mathrm{i}+\mathrm{n}, \quad 1 \leq \mathrm{i} \leq \mathrm{n}$ and

$\mathrm{f}\left(\mathrm{u}_{\mathrm{i}}{ }^{\prime \prime}\right)=\mathrm{w}_{1}$, when $\mathrm{i}=1$

\section{PERMUTATIONS LABELING OF}

\section{SOME SPLIT GRAPHS}

Theorem 3.1: $\operatorname{Spl}\left(\mathrm{P}_{\mathrm{n}}\right)$ is a permutation graph

Proof:

Let $\mathrm{u}_{1}, \mathrm{u}_{2}, \ldots, \mathrm{u}_{\mathrm{n}}$ be the vertices corresponding to $\mathrm{v}_{1}, \mathrm{v}_{2}, \ldots$, $v_{n}$ of $P_{n}$ which are added to obtain $\operatorname{Spl}\left(P_{n}\right)$. Let $G$ be the graph $\operatorname{Spl}\left(\mathrm{P}_{\mathrm{n}}\right)$. Then $|\mathrm{V}(\mathrm{G})|=2 \mathrm{n}$ and $|\mathrm{E}(\mathrm{G})|=3(\mathrm{n}-1)$. To define $\quad \mathrm{f:} V(\mathrm{G}) \rightarrow\{1,2, \ldots, 2 \mathrm{n}\}$ as follows.

$\mathrm{f}\left(\mathrm{v}_{\mathrm{i}}\right)=\mathrm{i} \quad(1 \leq \mathrm{i} \leq \mathrm{n})$

$\mathrm{f}\left(\mathrm{u}_{\mathrm{i}}\right)=\mathrm{n}+\mathrm{i} \quad(1 \leq \mathrm{i} \leq \mathrm{n})$

Hence $\operatorname{Spl}\left(\mathrm{P}_{\mathrm{n}}\right)$ is a permutation graph

Illustration: 3.2: Permutation labeling of the graph $\operatorname{Spl}\left(\mathrm{P}_{4}\right)$ is shown in Figure-6.

Illustration: 3.3: Permutation labeling of the graph $\operatorname{Spl}\left(\mathrm{P}_{7}\right)$ is shown in Figure-7.

$$
\begin{gathered}
\mathrm{f}\left(\mathrm{v}_{\mathrm{i}^{\prime}}\right)=\mathrm{i}+3 \mathrm{n}+1, \quad 1 \leq \mathrm{i} \leq \mathrm{n} \\
\mathrm{f}\left(\mathrm{v}_{\mathrm{i}^{\prime}}\right)=\mathrm{i}+2 \mathrm{n}+1, \quad 1 \leq \mathrm{i} \leq \mathrm{n} \\
\mathrm{f}\left(\mathrm{v}_{\mathrm{i}^{\prime \prime}}\right)=\mathrm{w}_{3}, \quad \text { when } \mathrm{i}=1 \text { and }
\end{gathered}
$$

\section{Illustration:2.9:}

The permutation labeling of a graph $\mathbf{G}$ obtained by joining two copies of shadow graphs $\mathbf{D}_{2}\left(\mathrm{C}_{5}\right)$ by a path of arbitrary length is shown in figure-5

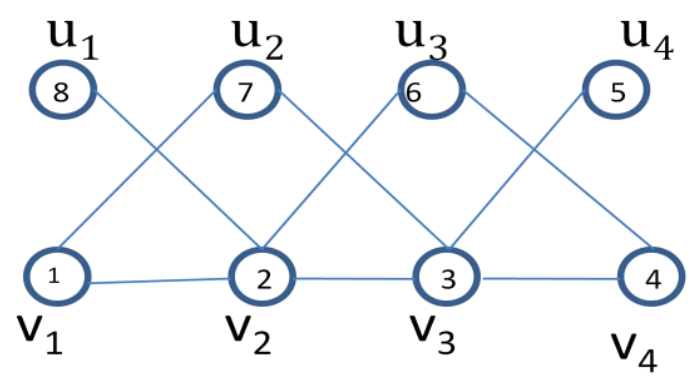

Fig-6: Permutation labeling of the graph $\operatorname{Spl}\left(\mathbf{P}_{4}\right)$ 


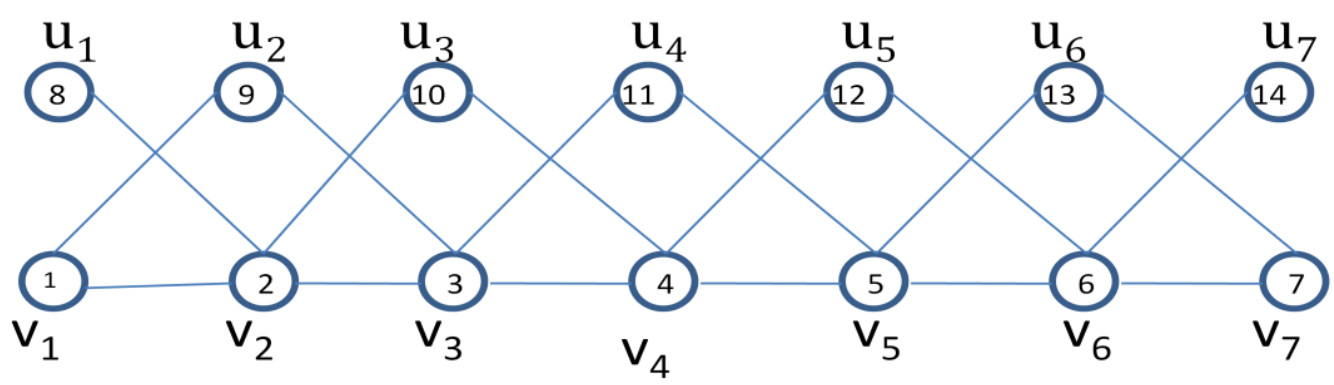

Fig-7: Permutation labeling of the graph $\operatorname{Spl}\left(\mathbf{P}_{7}\right)$.

Theorem 3.4: $\mathrm{Spl}\left(\mathrm{K}_{1, \mathrm{n}}\right)$ is a permutation graph.

Proof: Let $\mathrm{u}_{1}, \mathrm{u}_{2}, \ldots, \mathrm{u}_{\mathrm{n}}$ be the vertices corresponding to $\mathrm{v}_{1}$ $, v_{2}, \ldots, v_{n}$ of $P_{n}$ which are added to obtain $\operatorname{Spl}\left(P_{n}\right)$. Let $G$ be the graph $\operatorname{Spl}\left(\mathrm{P}_{\mathrm{n}}\right)$. Then $|\mathrm{V}(\mathrm{G})|=2 \mathrm{n}+2$ and $|\mathrm{E}(\mathrm{G})|=3 \mathrm{n}$. To define

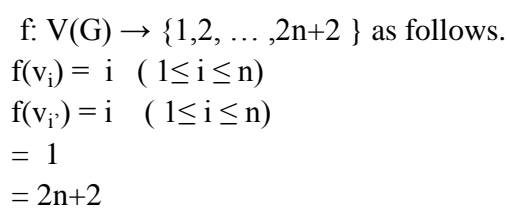

$=2 \mathrm{n}+2$

and all the edges of $\operatorname{Spl}\left(\mathrm{K}_{1, \mathrm{n}}\right)$ have different weights for the edges. Hence $\operatorname{Spl}\left(\mathrm{K}_{1, \mathrm{n}}\right)$ is a permutation graph.

Illustration:3.5: Permutation labeling of the graph

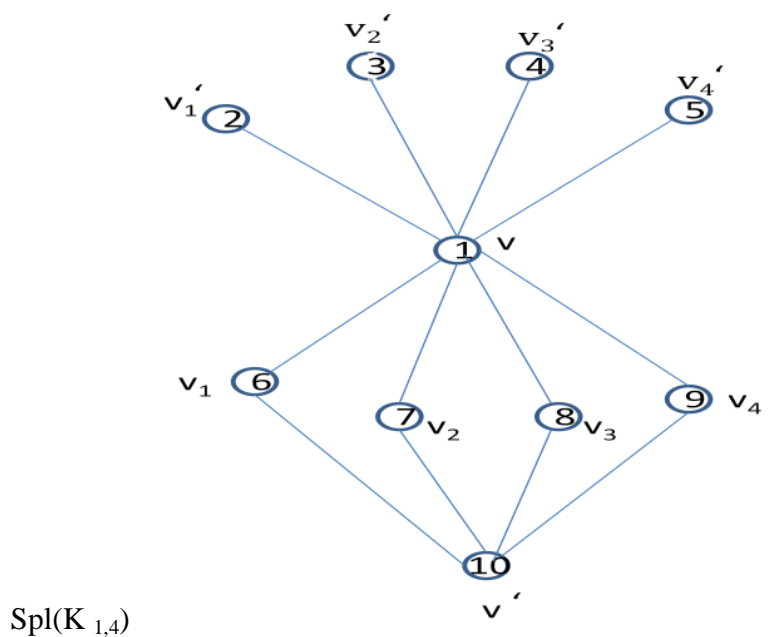

Fig-8:Permutation labeling of the graph $\operatorname{Spl}\left(K_{1,4}\right)$

\section{CONCLUSION}

Finding shadow and split graphs seems to be a useful tool to generate new permutation graphs. Here I investigated shadow graphs of path $\mathrm{P}_{\mathrm{n}}$, star $\mathrm{K}_{1, \mathrm{n}}$ and path union of shadow graphs of cycle $C_{n}$ are permutation graphs. Further I proved that the split graphs of path $\mathrm{P}_{\mathrm{n}}$ and star $\mathrm{K}_{1, \mathrm{n}}$ are permutation graphs.One can investigate further more graphs which satisfies permutation labeling.

\section{ACKNOWLEDGEMENTS}

The author is very thankful to the referees for their valuable comments and kind suggestions.

\section{REFERENCES}

[1] B.D.Acharya and S.M .Hegde, Arithmetic graphs, J.Graph theory, 14(3) (1990),275 - 299

[2] L.Beineke and S.M.Hegde, Strongly multiplicative graphs, Discuss. Math. Graph theory, 21(2001), 63- 75.

[3] D.M.Burton, Elementary Number theory, Second edition, Wm.C.Brown Company Publishers, 1980.

[4] Frank Harrary, Graph theory, Narosa Publishing House(2001).

[5] J A Gallian, A dynamic survey of graph labeling, The Electronics journal of Combinatories, 17(2010) \# DS6

[6] Gary Chartrnd, Ping Zhang,Introduction to Graph theory, McGRAW- Hill International Edition

[7] S.C Shee Y.S Ho , “ The cardinality of path union of $n$ copies of a graph “, Discrete Math, 151 (1996), 221- 229

[8] Suresh Manjanath Hegde , Sudhakar Shetty, Combinatorial labeling of graphs ,Applied Mathematics E- notes,6(2006), 251-258@ Revised $11^{\text {th }}$ September

[9] J. Shiama" Square sum labeling for some middle and total graphs" International Journal of Computer Applications (0975-08887) Volume 37- No.4 January 2012

[10] J. Shiama "Permutation labeling for some split graphs" Proceedings of the National Conference on Mathematical Modelling and Simulation. NCMS' $1211^{\text {th }}$ Feb.2012

[11] D B West, Introduction to Graph Theory, Prentice-Hall, India, 2001 